\title{
ASYMPTOTIC BEHAVIOR OF NONLINEAR DIFFUSIONS
}

\author{
Manuel Del Pino and Jean Dolbeault
}

\begin{abstract}
We describe the asymptotic behavior as $t \rightarrow \infty$ of the solution of $u_{t}=\Delta_{p} u$ in $\mathbb{R}^{N}$, for $(2 N+1) /(N+1) \leq p<N$ and non-negative, integrable initial data. Optimal rates in $L^{q}, q=2-1 /(p-1)$ for the convergence towards a self-similar profile corresponding to a solution with Dirac distribution initial data are found. They are connected with optimal constants for a Gagliardo-Nirenberg inequality.
\end{abstract}

\section{Introduction and main result}

Let us consider a non-negative solution $u(x, t)$ of the $p$-Laplace flow in $\mathbb{R}^{N}$, namely the equation

$$
u_{t}=\nabla \cdot\left(|\nabla u|^{p-2} \nabla u\right)
$$

for a given initial data $u(x, 0)=u_{0}(x) \geq 0$, where $p>1$ and $N \geq 2$. Such a solution exists and it is unique in the natural weak sense under rather mild conditions on $u_{0}$. In particular, this is the case for $u_{0} \in L^{1}$, condition which we will assume in what follows. We refer the reader to $[11,10]$ for general results on the issues of existence, uniqueness and regularity of non-negative solutions.

It is known that a fundamental solution of (1) exists whenever $p>2 N /(N+1)$. This is a non-negative solution of (1) which takes a Dirac mass $M \delta_{0}, M>0$, as initial data. It is unique, see [14], and explicitly given by

$$
u_{\infty}(x, t)=(\gamma t)^{-N / \gamma} v_{\infty}\left((\gamma t)^{-1 / \gamma} x\right)
$$

where

$$
v_{\infty}(x)=\left(C-\frac{p-1}{p}(q-1)|x|^{\frac{p}{p-1}}\right)_{+}^{\frac{1}{q-1}},
$$

with $q=2-1 /(p-1), \quad \gamma=(N+1) p-2 N$. The constant $C$ is uniquely determined from the initial mass $M$, which is preserved in time. The importance of these solutions arises from the fact that they determine at first order the long-time behavior of solutions to (1) which have finite mass. This has been established in [14], in the following sense: if $u$ is a non-negative solution of (1) with $u_{0} \in L^{1}$, then

$$
\lim _{t \rightarrow \infty}\left\|u(\cdot, t)-u_{\infty}(\cdot, t)\right\|_{1}=0=\lim _{t \rightarrow \infty} t^{N / \gamma}\left\|u(\cdot, t)-u_{\infty}(\cdot, t)\right\|_{\infty} .
$$

Recieved September 18, 2001. 
Notations. We shall note $M=\int u_{0} d x$. Throughout this paper, for any $c>0$, $\|v\|_{c}=\left(\int|v|^{c} d x\right)^{1 / c}$. Integrals are taken over $\mathbb{R}^{N}$ and $L^{s}$ means $L^{s}\left(\mathbb{R}^{N}\right)$ unless it is explicitely specified.

Estimates (3) tell us that the difference between $u$ and the fundamental solution $u_{\infty}$ goes to zero in $L^{1}$ and uniformly faster than $u_{\infty}$ itself. Let us rephrase this result in a convenient form for our purpose: the mass of the solution $u(x, t)$ is preserved in time but spreads so that the solution itself decays in $L^{\infty}$-norm as $O\left(t^{-N / \gamma}\right)$. However a scaling brings the solution into a function converging to a universal profile $v_{\infty}$. Such an asymptotic regime of $u(\cdot, t)$ as $t \rightarrow \infty$ is usually referred as intermediate asymptotics in the literature.

Let us be more precise. Consider the function

$$
R(t)=(1+\gamma t)^{1 / \gamma}
$$

and the function $v$ defined, with $R=R(t)$, through the change of variables

$$
u(x, t)=R^{-N} v\left(R^{-1} x, \log R\right)
$$

Since $R$ satisfies $\frac{d R}{d t}=R^{-(N+1)(p-1)+N}, v(x, \tau)$ then solves the equation

$$
v_{\tau}=\nabla \cdot\left(|\nabla v|^{p-2} \nabla v\right)+\nabla \cdot(x v)
$$

with the same initial data $u_{0}$ as $u$ and $\tau(t)=\log R(t)$. Let us then observe that $v_{\infty}$ given by (2) is precisely the positive radial (decreasing) solution of

$$
\nabla \cdot\left(\left|\nabla v_{\infty}\right|^{p-2} \nabla v_{\infty}\right)+\nabla \cdot\left(x v_{\infty}\right)=0
$$

with $\int v_{\infty} d x=M=\int u_{0} d x$.

Since $R(t) \sim(\gamma t)^{-1 / \gamma}$ as $t \rightarrow \infty$, relations (3) simply read

$$
\lim _{\tau \rightarrow \infty}\left\|v(\cdot, \tau)-v_{\infty}\right\|_{1}=0=\lim _{\tau \rightarrow \infty}\left\|v(\cdot, \tau)-v_{\infty}\right\|_{\infty},
$$

that is the convergence in $L^{1}$ and $L^{\infty}$ senses of $v$ towards the unique steady state $v_{\infty}$ with same mass as $v(\cdot, t)$. Of course, by interpolation,

$$
\lim _{\tau \rightarrow \infty}\left\|v(\cdot, \tau)-v_{\infty}\right\|_{s}=0 \quad \text { for all } 1<s<\infty,
$$

which means in the original variables $\left\|u(\cdot, t)-u_{\infty}(\cdot, t)\right\|_{s}=O\left(t^{-N(s-1) /(\gamma s)}\right)$. No result seems so far to be known concerning the rate at which convergences (5) take place. Our main result partly responds to this question, providing exponential rates of convergence when $p$ is further restricted. The following number comes into play

$$
\alpha=\left(1-\frac{1}{p}(p-1)^{(p-1) / p}\right) \frac{p}{p-1} .
$$

Theorem 1. Assume that $N \geq 2,(2 N+1) /(N+1) \leq p<N$. Let $\alpha$ be given by (6), $q=2-1 /(p-1)$ and consider a solution of (4) corresponding to a nonnegative $L^{1}$ initial data $u_{0}$ such that $L_{0}=\int\left(u_{0}^{q-1}+|x|^{p /(p-1)}\right) u_{0} d x$ is finite. 
(i) Let $p<2$. Then there exists a constant $K>0$ such that

$$
\left\|v(\cdot, \tau)-v_{\infty}\right\|_{q} \leq K e^{-\alpha \tau / 2} \quad \forall \tau>0 .
$$

(ii) Let $p>2$. Then there exists a constant $K>0$ such that

$$
\left\|v^{q}(\cdot, \tau)-v_{\infty}^{q}\right\|_{1 / q} \leq K e^{-\alpha \tau / 2} \quad \forall \tau>0 .
$$

Here $v_{\infty}$ is the unique stationary, non-negative, radial solution of (4) with mass $M$. The constants $K$ in (i) and (ii) only depend on $p, N, M$ and $L_{0}$.

Coming back to the original variables and exploiting the asymptotic behaviour of $R$, we get the following result on the intermediate asymptotics of $u$.

Corollary 1. Under the same assumptions on $N, p$ and $u_{0}$ as in Theorem 1,

$$
\begin{aligned}
\left\|u(\cdot, t)-u_{\infty}(\cdot, t)\right\|_{q}=O\left(t^{-(\eta+N(q-1) /(\gamma q))}\right), & \text { if } p<2, \\
\left\|u^{q}(\cdot, t)-u_{\infty}^{q}(\cdot, t)\right\|_{1 / q} \leq O\left(t^{-(\eta+N(1-q) q / \gamma)}\right), & \text { if } p>2,
\end{aligned}
$$

as $t \rightarrow \infty$, where $\eta=\frac{N \alpha}{2 \gamma}=\frac{p-(p-1)^{(p-1) / p}}{2(p-1)((N+1) p-2 N)}$.

These estimates also provide new power decay rates for other norms $L^{s}$ through interpolation with relations (3), at least if $u_{0}$ is bounded in $L^{\infty}$.

The rest of this paper is devoted to a proof of Theorem 1. For that purpose, we define a natural Lyapunov, or entropy, functional. A result on optimal constants for a Gagliardo-Nirenberg inequality [9] provides an exponential rate of convergence with optimal exponent, when the convergence is measured in terms of the entropy. The conclusion then follows from a control of the distance to the stationary solution by the entropy itself. A similar strategy has been used in the case of the porous medium equation $u_{t}=\Delta u^{m}$ in $[7,8]$, in detecting rates of convergence to a fundamental solution in $L^{1}$ (in $L^{\infty}$, this phenomenon was first studied in [12] - also see [17, 18]). A different approach, based on the so-called entropy-entropy production method (see for instance [2] for linear diffusions, and $[5,16, ?, 13,15]$ for nonlinear diffusions) has recently been developed. This approach has established, for the solution of (1) and related equations, decay estimates similar to the ones of the fundamental solution, but has apparently not succeeded to catch intermediate asymptotics in the case of the $p$-Laplacian operator.

\section{Exponential decay of the entropy}

Using the notation introduced in the first section, and assuming $q=2-1$ / $(p-1) \neq 1$, we consider the convex functional

$$
E[w]=\int\left(\sigma(w)-\sigma\left(v_{\infty}\right)-\sigma^{\prime}\left(v_{\infty}\right)\left(w-v_{\infty}\right)\right) d x
$$


where $\sigma$ is defined on $(0, \infty)$ by $\sigma(s)=\left(s^{q}-1\right) /(q-1)$. Here, we recall that $v_{\infty}$ is defined by (2) with $C$ chosen so that $\int v_{\infty} d x=\int u_{0} d x$. Note that using properties of the Gamma function, $C$ can be explicitly computed according to

$$
\log M=A \log C-N^{*} \log \frac{p-1}{p}+\log \left(B \pi^{\frac{N}{2}} \Gamma\left(N^{*}+1\right) / \Gamma\left(\frac{N}{2}+1\right)\right)
$$

with $N^{*}=\frac{p-1}{p} N, A=\left(N^{*}+\frac{1}{q-1}\right), B=\Gamma\left(\frac{q}{q-1}\right) / \Gamma\left(N^{*}+\frac{q}{q-1}\right)$ if $p<2$, and $B=\Gamma\left(\frac{1}{1-q}-N^{*}\right) / \Gamma\left(\frac{1}{1-q}\right)$ if $p>2$.

The term $\sigma^{\prime}\left(v_{\infty}\right)$ is explicitely given by: $q C /(q-1)-q(p-1)|x|^{p /(p-1)} / p$. Let $v(\tau, x)$ be the solution of (4) with initial data $u_{0}$ and denote

$$
\Sigma(\tau)=E[v(\cdot, \tau)],
$$

which we call the entropy of the solution, in analogy to similar objets arising in kinetic theory. We introduce the number

$$
\kappa_{p}=\frac{1}{p}(p-1)^{(p-1) / p} .
$$

Observe that $\alpha$ defined in (6) can be written $\alpha=\left(1-\kappa_{p}\right) p /(p-1)$. The main step in the proof of Theorem 1 is the following result.

Proposition 1. Under the assumptions of Theorem 1 and with the above notations, any solution $v$ with initial data $u_{0}$ satisfies

$$
\Sigma(\tau) \leq e^{-\alpha \tau} \cdot \Sigma(0) \quad \forall \tau>0
$$

The proof of this result is a consequence of two estimates which we present in the remaining of this section. Let us first observe that

$$
\begin{aligned}
\frac{d \Sigma}{d \tau} & =-\int \nabla\left(\sigma^{\prime}(v)-\sigma^{\prime}\left(v_{\infty}\right)\right) \cdot\left(|\nabla v|^{p-2} \nabla v+x v\right) d x \\
& =-q\left(I_{1}+I_{2}+I_{3}+I_{4}\right)
\end{aligned}
$$

where

$$
\begin{aligned}
I_{1} & =\int v^{q-2}|\nabla v|^{p} d x & I_{2} & =\int|x|^{\frac{p}{p-1}} v d x \\
I_{3} & =-\frac{N}{q} \int v^{q} d x & I_{4} & =\int|\nabla v|^{p-2} \nabla v \cdot|x|^{\frac{1}{p-1}-1} x d x
\end{aligned}
$$

Using Hölder's inequality, we get the estimate

$$
\left.\left|\int\right| \nabla v\right|^{p-2} \nabla v \cdot|x|^{\frac{1}{p-1}-1} x d x \mid \leq\left(\int v^{q-2}|\nabla v|^{p} d x\right)^{\frac{p-1}{p}}\left(\int|x|^{\frac{p}{p-1}} v d x\right)^{\frac{1}{p}}
$$

and thus obtain $\left|I_{4}\right| \leq \kappa_{p}\left(I_{1}+I_{2}\right)$ where $\kappa_{p}$ is the number given by (8), since $\kappa_{p}(1+t)-t^{1 / p} \geq 0$ for any $t \geq 0$. Now, identity (9) and estimate (1) directly yield the following result. 
Lemma 1. For any measurable function $v$,

$$
I_{1}+I_{2}+I_{3}+I_{4} \geq\left(1-\kappa_{p}\right)\left(I_{1}+I_{2}\right)+I_{3}
$$

with $\kappa_{p}$ and $\left(I_{1}, I_{2}, I_{3}, I_{4}\right)$ respectively defined by (8) and (10).

The second estimate we require is the following.

Lemma 2. Assume that $N>1,(2 N+1) /(N+1) \leq p<N$. Then for any function $v$ for which all integrals make sense and are finite,

$$
E[v] \leq \frac{p-1}{p}\left(I_{1}+I_{2}+\frac{I_{3}}{1-\kappa_{p}}\right)
$$

where $E, \kappa_{p}$ and $\left(I_{1}, I_{2}, I_{3}\right)$ are respectively given by (7), (8) and (10).

Proof. For the proof of this estimate, we invoke an optimal Gagliardo-Nirenberg inequality found in [9].

Lemma 3. Let $1<p<N, 1<a \leq \frac{p(N-1)}{N-p}$, and $b=p \frac{a-1}{p-1}$. There exists a positive constant $\mathcal{S}$ such that for any function $w \in W_{\mathrm{loc}}^{1, p}$ with $\|w\|_{a}+\|w\|_{b}<\infty$,

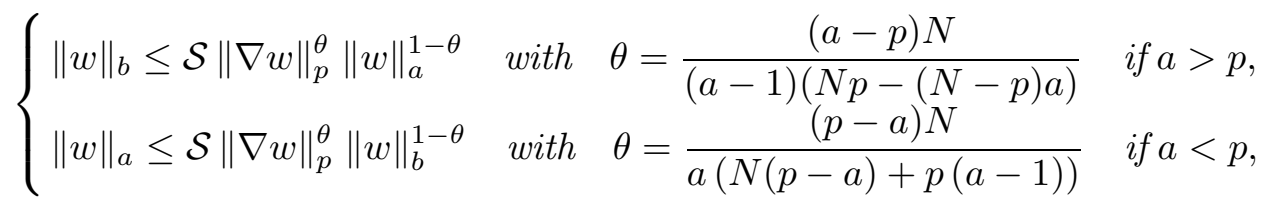

and equality holds for any function taking, up to a translation, the form

$$
\bar{w}(x)=A\left(1+B|x|^{\frac{p}{p-1}}\right)_{+}^{-(p-1) /(a-p)},
$$

for some $(A, B) \in \mathbb{R}^{2}$, where $B$ has the sign of $a-p$.

With the special choice $b=\frac{p(p-1)}{p^{2}-p-1}, a=b q$, we can rephrase Lemma 3 into a single non homogeneous inequality for $v=w^{b}$. For $p \neq 2$, let

$$
\mathcal{F}[v]=\int v^{-\frac{1}{p-1}}|\nabla v|^{p} d x-\frac{1}{q}\left(\frac{N}{1-\kappa_{p}}+\frac{p}{p-2}\right) \int v^{q} d x,
$$

with $\kappa_{p}$ defined by (8). If $\frac{2 N+1}{N+1} \leq p<N$, then

$$
\mathcal{F}[v] \geq \mathcal{F}\left[v_{\infty}\right]
$$

where $v_{\infty}$ is defined as in (2) with $C$ so that $\|v\|_{1}=\left\|v_{\infty}\right\|_{1}$. In fact, let us notice first that $\frac{N}{1-\kappa_{p}}+\frac{p}{p-2}$ is positive for $p>2$ and negative for $p<2$. One can perform a scaling leaving $\|v\|_{1}$ invariant in the first case, $\|v\|_{q}$ in the second case. This reduces the inequality for $v$ to one of the cases of Lemma 3 . The result follows by identification of a minimizer. This concludes the proof of Lemma 2 .

Proposition 1 is a direct consequence of Lemmas 1 and 2:

$$
\Sigma \leq \frac{q}{1-\kappa_{p}} \frac{p-1}{p}\left[\left(1-\kappa_{p}\right)\left(I_{1}+I_{2}\right)+I_{3}\right] .
$$

Observe in particular that $\kappa_{p}<1$ for any $p>1$. 


\section{Proof of Theorem 1}

We are going to use the result of Proposition 1 in order to prove Theorem 1. In order to relate the exponential decay of the entropy with that of $L^{s}$-norms, we require a form of the so-called Csiszár-Kullback inequality corresponding to $\sigma(s)=\left(s^{q}-1\right) /(q-1)$. Although this is a rather standard fact, see for instance $[3,6]$, for completeness we provide a proof which is extracted from [6] (also see $[1, ?])$.

Lemma 4. Let $f$ and $g$ be two nonnegative functions in $L^{q}(\Omega)$ for a given domain $\Omega$ in $\mathbb{R}^{N}$. Assume that $q \in(1,2]$. Then

$$
\int_{\Omega}\left[\sigma\left(\frac{f}{g}\right)-\sigma^{\prime}(1)\left(\frac{f}{g}-1\right)\right] g^{q} d x \geq \frac{q}{2} \frac{\|f-g\|_{L^{q}(\Omega)}^{2}}{\max \left(\|f\|_{L^{q}(\Omega)}^{2-q},\|g\|_{L^{q}(\Omega)}^{2-q}\right)} .
$$

Proof. A second order Taylor expansion around 1 gives

$$
\Sigma=\int_{\Omega} \sigma\left(\frac{f}{g}\right) g^{q} d x=\frac{1}{2} \int_{\Omega} \sigma^{\prime \prime}\left(1+\theta\left(\frac{f}{g}-1\right)\right) g^{q}\left(\frac{f}{g}-1\right)^{2} d x,
$$

for some function $\theta$ with values in [0,1]. On the subdomains of $\Omega$ corresponding to $f<g$ and $f>g$, it happens that, respectively, $\sigma^{\prime \prime}(1+\theta(f / g-1))$ and $\sigma^{\prime \prime}(1+\theta(f / g-1))(g / f)^{q-2}$ are both bounded from below by $\sigma^{\prime \prime}(1)$. Thus

$$
\Sigma \geq \frac{q}{2}\left(\int_{f<g} \frac{|f-g|^{2}}{g^{2-q}} d x+\int_{f>g} \frac{|f-g|^{2}}{f^{2-q}} d x\right) .
$$

Hölder's inequality applied to the first term gives

$$
\int_{f<g} \frac{|f-g|^{q}}{g^{q\left(1-\frac{q}{2}\right)}} \cdot g^{q\left(1-\frac{q}{2}\right)} d x \leq\left(\int_{f<g} \frac{|f-g|^{2}}{g^{2-q}} d x\right)^{\frac{q}{2}} \cdot\left(\int_{f<g} g^{q} d x\right)^{1-\frac{q}{2}},
$$

and a similar result holds on the subdomain corresponding to $f>g$.

Proof of Theorem 1. Combining Proposition 1 if $q>1$ and Lemma 4, part (i) of the theorem follows since $\left\|v_{\infty}(\cdot, \tau)\right\|_{q}^{q-2}$ is certainly bounded in terms of $\Sigma(0)$. On the other hand, for $q<1$, we may write

$$
\sigma(s)-\sigma^{\prime}(1)(s-1)=\frac{\left(s^{q}\right)^{\frac{1}{q}}-1-\frac{1}{q}\left(s^{q}-1\right)}{\frac{1}{q}-1},
$$

and apply Lemma 4 with $q$ replaced by $1 / q$. The result of part (ii) then follows from Proposition 1, and the proof is completed.

\section{Acknowledgements}

This research has been supported by ECOS-Conicyt under contract C98E03. The first and the second author have been partially supported respectively by Grants FONDAP and Fondecyt Lineas Complementarias 8000010, and by European TMR-Network ERBFMRXCT970157 and the C.M.M. (UMR CNRS no. 2071), Universidad de Chile. 


\section{References}

[1] A. Arnold, P. Markowich, G. Toscani, and A. Unterreiter, On generalized Csiszár-Kullback inequalities, Monatsh. Math. 131 (2000), 235-253.

[2] _ On convex Sobolev inequalities and the rate of convergence to equilibrium for Fokker-Planck type equations, Comm. Partial Differential Equations 26 (2001), 43-100.

[3] M. J. Cáceres, J. A. Carrillo, and J. Dolbeault, Nonlinear stability in $L^{p}$ for a confined system of charged particles, SIAM J. Math. Anal. 34 (2002), 478-494.

[4] J. A. Carrillo, A. Jüngel, P. A. Markowich, G. Toscani, and A. Unterrreiter, Entropy dissipation methods for degenerate parabolic problems and generalized Sobolev inequalities, Monatsh. Math. 133 (2001), 1-82.

[5] J.A. Carrillo and G. Toscani, Asymptotic $L^{1}$-decay of solutions of the porous medium equation to self-similarity, Indiana Univ. Math. J. 49 (2000), 113-142.

[6] C. Cid and J. Dolbeault, Defocusing nonlinear Schrödinger equation: confinement, stability and asymptotic stability, in preparation.

[7] M. Del Pino and J. Dolbeault, Generalized Sobolev inequalities and asymptotic behaviour in fast diffusion and porous media problems, Preprint Ceremade no. 9905.

[8] _ Best constants for Gagliardo-Nirenberg inequalities and application to nonlinear diffusions, J. Math. Pures Appl. (9) 81 (2002), 847-875.

[9] _ The optimal Euclidean $L^{p}$-Sobolev logarithmic inequality, to appear in J. Funct. Anal.

[10] E. Di Benedetto, Degenerate parabolic equations, Springer-Verlag, New York, 1993.

[11] E. Di Benedetto and M. A. Herrero, On the Cauchy problem and initial traces for a degenerate parabolic equation, Trans. Amer. Math. Soc. 314 (1989), 187-224.

[12] A. Friedmann and S. Kamin, The asymptotic behaviour of gas in a $n$-dimensional porous medium, Trans. Amer. Math. Soc. 262 (1980), 551-563.

[13] A. Jüngel, P. A. Markowich and G. Toscani, Decay rates for solutions of degenerate parabolic equations, Proceedings of the USA-Chile Workshop on Nonlinear Analysis (Via del Mar-Valparaiso, 2000), 189-202. Electron. J. Differ. Equ. Conf., 6, Southwest Texas State Univ., San Marcos, TX, 2001.

[14] S. Kamin and J. L. Vázquez, Fundamental solutions and asymptotic behaviour for the p-Laplacian equation, Rev. Mat. Iberoamericana 4 (1988), 339-354.

[15] C. Lederman and P. A. Markowich, On fast-diffusion equations with infinite equilibrium entropy and finite equilibrium mass, to appear in Comm. Partial Differential Equations.

[16] F. Otto, The geometry of dissipative evolution equations: the porous medium equation, Comm. Partial Differential Equations 26 (2001), 101-174.

[17] J. L. Vázquez, Asymptotic behaviour of nonlinear parabolic equations. Anomalous exponents, Degenerate diffusions (Minneapolis, MN, 1991), 215-228, IMA Vol. Math. Appl., 47, Springer, New York, 1993.

[18] _ Asymptotic behaviour for the Porous Medium Equation in the whole space, to appear in Journal of Evolution Equations 3 (2003), 67-118.

D.I.M. And C.M.M. (UMR CNRS no. 2071), Universidad de Chile, Casilla 170, Correo 3, Santiago, Chile.

E-mail address: delpino@dim.uchile.cl

CEREMAde (UMR CNRS no. 7534), Université Paris IX-Dauphine, Place de LatTre de TASSIGny, 75775 PARIS CÉdeX 16, France.

E-mail address: dolbeaul@ceremade.dauphine.fr 